\title{
BMJ Open Gestational diabetes prevention and treatment: a protocol for developing core outcome sets
}

\author{
Aoife Maria Egan (D) , ${ }^{1}$ Fidelma P Dunne, ${ }^{2}$ Linda M Biesty, ${ }^{2,3}$ Delia Bogdanet, ${ }^{2}$ \\ Caroline Crowther, ${ }^{4}$ Eugene Dempsey, ${ }^{5}$ Shakila Thangaratinam, ${ }^{6}$ Declan Devane, ${ }^{2,7}$ \\ Narjes Fhelelboom, ${ }^{2}$ On behalf of the INSPIRED (INternational collaboration for \\ Studies in PREgnacy and Diabetes) research group.
}

To cite: Egan AM, Dunne FP, Biesty LM, et al. Gestational diabetes prevention and treatment: a protocol for developing core outcome sets. BMJ Open 2019;9:e030574. doi:10.1136/ bmjopen-2019-030574

- Prepublication history and additional material for this paper are available online. To view these files, please visit the journal online (http://dx.doi. org/10.1136/bmjopen-2019030574).

Received 20 March 2019 Revised 17 September 2019 Accepted 17 October 2019

Check for updates

(C) Author(s) (or their employer(s)) 2019. Re-use permitted under CC BY-NC. No commercial re-use. See rights and permissions. Published by BMJ.

For numbered affiliations see end of article.

Correspondence to Dr Aoife Maria Egan; egan.aoife@mayo.edu

\section{ABSTRACT}

Introduction Selective reporting bias, inconsistency in the chosen outcomes between trials and irrelevance of the chosen outcomes for women, limit the efficiency and value of research for prevention and treatment of gestational diabetes mellitus (GDM). One way to address these challenges is to develop core outcome sets (COSs). Methods and analysis The aim of this manuscript is to present a protocol for a study to develop COSs for GDM prevention and treatment. This is a three-phase project consisting of (1) a systematic review of the literature to create two lists of outcomes that have been reported in trials and systematic reviews of trials of interventions for the prevention and treatment of GDM, (2) a three-round, web-based e-Delphi survey with key stakeholders to prioritise these outcomes and (3) a consensus meeting to resolve any remaining disagreements and to agree on two COSs.

Ethics and dissemination Ethical approval to conduct this study was obtained from the ethics committee at Galway University Hospitals on 13 December 2018 (Reference: C.A.2078). We will disseminate our research findings through peer-reviewed, open access publications and present at international conferences to reach a wide range of knowledge users.

\section{INTRODUCTION}

Gestational diabetes mellitus (GDM) is the occurrence of glucose intolerance during pregnancy and usually resolves after birth. ${ }^{1}$ It is one of the major conditions contributing to maternal morbidity and fetal and neonatal mortality and morbidity. There is recognition of increasing prevalence of GDM worldwide, with prevalence ranging between $1 \%$ and $16.9 \%$ across countries depending on the criteria of diagnosis. $^{23}$

GDM is associated with short-term and long-term complications for the mother and her infant with wider health and economic burden. ${ }^{4-14}$ The aim of GDM management is to optimise maternal glycaemic control and consequently improve pregnancy outcomes. ${ }^{15}$ Lifestyle advice and dietary interventions

\section{Strengths and limitations of this study}

- Systematic review and core outcome set (COS) development methods that adhere to Core Outcome Measures in Effectiveness Trials (COMET) guidelines.

- Plans to engage with multiple stakeholders to generate diverse viewpoints with worldwide representation.

- Key study staff representing international collaboration.

- This study will not involve a qualitative dimension and the study components will be limited to the English language.

- As a repository of definitions already exists, this study will not address how to measure the outcomes included in the final COSs.

are usually recommended as the primary therapeutic strategy for women with GDM. If this strategy is insufficient, oral hypoglycaemic medications or insulin therapy can be added to achieve target glycaemic control. ${ }^{15}$ However, treatment recommendations differ between, and also within, countries.

The burden of GDM has resulted in an increase in randomised trials of interventions for the prevention and treatment of GDM in recent years. For instance, MEDLINE included $<1700$ citations indexed as 'gestational diabetes mellitus' before 2000, compared with >3000 citations between 2001 and $2010 .^{16}$ In addition, the majority of randomised trials on GDM have been published in the last 10 years. ${ }^{16}$

Heterogeneity in the outcomes reported in the trials makes combining and comparing results between trials difficult. ${ }^{17}$ Consequently, this may limit the validity of metaanalysis and reliability of evidence. A recent Cochrane review by Brown et al (2017) of 15 trials (4501 women, 3768 infants) evaluating the effectiveness of combined lifestyle 
interventions, with or without pharmacotherapy, for treating women with GDM, identified the main methodological limitation as the inconsistency in outcome reporting in the included trials. ${ }^{15}$ A recent systematic review, evaluating the effects of oral antidiabetic medications for treating women with GDM, included 11 studies (1487 women and their offspring), and stated that there was substantial heterogeneity in the outcomes reported across trials included in the review. ${ }^{18}$

Selective reporting bias occurs when additional outcomes that were not prespecified are reported; or where outcomes that had been prespecified are not reported. For example, a randomised trial of a carbose versus placebo in the treatment of GDM reported many more maternal and neonatal outcomes in the published paper than were prespecified in the trial registration document. ${ }^{19}$ Fully informed decisions about care cannot be made based on research with selective reporting of outcomes as this could lead to use of ineffective and sometimes harmful interventions, and under use of interventions that might be effective. This means selection of results that cover only a fraction of relevant evidence can lead to inappropriate decisions, for example, prescribing ineffective treatment or not prescribing effective treatment.

Last, outcomes reported in literature may not reflect the values and preferences of study participants. For findings to influence policy and practice, outcomes need to be relevant and important to key stakeholders and, in particular, women. One study on women's attitudes and perspectives on dietary advice as a strategy in the management of GDM found that stress and anxiety were reported as important outcomes following dietary advice from the perspective of the women. ${ }^{20}$ These outcomes are rarely reported in the existing GDM management literature. Using relevant outcomes for women and other stakeholders in GDM, this research will enhance pregnancy care and improve maternal and offspring health.

The development of core outcome sets (COSs) for evaluating the effectiveness of interventions for the prevention and treatment of GDM would help minimise heterogeneity in outcomes reported, maximise potential for synthesising of information and ensure that all stakeholders' perspectives are represented in the outcomes reported. The COS represents the minimum set of outcomes that are expected to always be measured and reported, though, trialists are not restricted to these set of outcomes as they can measure additional outcomes. At the outset, potential barriers to COS implementation should be considered, and in this particular scenario, generating a list of key outcomes with worldwide relevance may be challenging.

The Core Outcome Measures in Effectiveness Trials (COMET) handbook ${ }^{21}$ and the Core Outcome SetSTAndards for Development (COS-STAD) $2^{2}$ brings together the current thinking and methodological research on this subject and provides minimal standards to be followed by COS developers. Relevant to the subject of GDM, the Core Outcomes in Women's and Newborn Health initiative advocates for the development of COSs in this area and has been endorsed by the editors of over 80 scientific journals. ${ }^{23}$

\section{Aims and objectives \\ Aims}

To present a protocol for a study to develop two COSs - one for trials evaluating interventions for the prevention of GDM and another for trials evaluating interventions for treatment of GDM.

\section{Objectives}

1. To conduct a systematic review to identify two comprehensive lists of outcomes used previously in trials evaluating the effectiveness of interventions for both the prevention and treatment of GDM.

2. Prioritisation and generation of two lists of outcomes based on the preferences of relevant stakeholder groups using e-Delphi methodology.

3. Achieve a consensus on two COSs through a face-toface meeting.

\section{Scope of the COS}

The first COS will be developed for effectiveness trials evaluating any intervention for prevention of GDM, and the second COS will be developed for effectiveness trials evaluating any intervention for treatment of GDM.

Other forms of diabetes mellitus are outside the scope of these COSs.

\section{Study oversight}

An international study advisory group (SAG) has been established. The SAG includes representatives from researchers $(n=2)$, healthcare professionals (an endocrinologist and an obstetrician $),(n=2)$ and women with a previous diagnosis of GDM $(n=2)$. The remit of the SAG is to oversee the study, provide feedback on the study protocol and list of outcomes, assist with circulation of the e-Delphi survey and to contribute to the final consensus meeting and distribution of the COSs.

\section{METHODS AND ANALYSIS}

The development of COSs will be conducted within three discrete work packages (figure 1) in keeping with COMET guidance and COS-STAD recommendations ${ }^{21}{ }^{22}$ :

1. A systematic review of the literature to identify outcomes that have been reported in trials and systematic reviews of trials of interventions for the prevention and treatment of GDM.

2. A three-round, web-based, e-Delphi survey with key stakeholders to prioritise outcomes.

3. A consensus meeting to finalise the COSs.

Patient and public involvement (PPI)

This study will be supported by PPI and both the core study team and the SAG containing patient representatives. It is anticipated that multiple patient representatives 


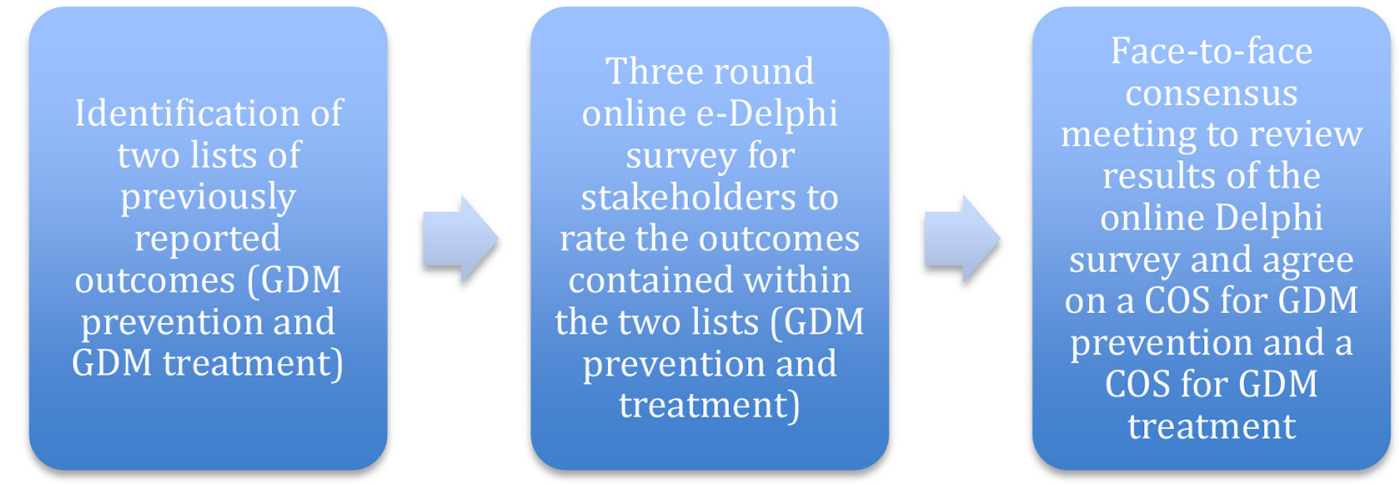

Figure 1 Overview of the COS development process. COS, core outcome set; GDM, gestational diabetes mellitus.

will participate in the e-Delphi survey and the consensus meeting.

\section{Systematic review of trials and systematic reviews of trials}

To identify outcomes used previously in trials, we will perform a systematic review of randomised trials and systematic reviews of randomised trials evaluating the effectiveness of interventions for the prevention and treatment of GDM. The systematic review will be performed in stages until outcome saturation is reached. A search will be performed for eligible studies from 2015 to 2019. Outcomes from 2017 to 2019 studies will be indexed initially and then outcomes from 2016 studies will be added. If no additional unique outcomes are identified from the 2016 studies, outcome saturation will be reached. If outcome saturation is not reached, outcomes from the 2015 studies will be added. If outcome saturation is still not reached, the search will be extended to include studies from 2013 to 2014 and so forth until outcome saturation is reached. Examining all available literature without a time window is resource intensive and may not yield important additional outcomes.

\section{Search strategy}

Using a comprehensive search strategy, the following databases will be searched for relevant studies: MEDLINE, Embase, Web of Science, Cochrane Library and CINAHL. Key terms used to guide the search will include 'GDM', 'gestational diabetes', 'maternal diabetes', 'hyperglycemia', 'pregnancy', 'prevention' and 'treatment', combined as appropriate using the Boolean operands 'AND' and 'OR'. Online supplementary appendix S1 outlines a sample search strategy. The reference lists of all relevant studies will be searched for additional relevant studies not retrieved from the electronic database search. Selection of articles will be restricted to English language publications.

\section{Types of studies}

We will include randomised trials and systematic reviews of randomised trials (with and without meta-analyses) comparing the effectiveness of various interventional strategies for both prevention and treatment of GDM.

\section{Types of interventions}

1. Pharmacological interventions for prevention and treatment of GDM.

2. Non-pharmacological interventions for prevention and treatment of GDM.

\section{Types of participants}

Participants will be women over the age of 16 years who are:

1. Diagnosed with GDM or

2. At risk of GDM. Women at risk of developing GDM include those with a family history of diabetes, overweight or having obese body mass index, previous GDM and high-risk ethnicity. ${ }^{24}$

\section{Assessment for eligibility}

The titles and abstracts of the retrieved studies will be screened and reviewed by two reviewers independently. Full texts of potential studies will be assessed for eligibility. In case of uncertainty regarding inclusion at title and abstract screening, a third reviewer will be consulted.

\section{Data extraction}

The following data will be extracted from each study: study design, author details, year and journal of publication, targeted condition, criteria for diagnosis of GDM, interventions under investigation and all outcomes as reported in the trial (including definitions, tools for measurement, thresholds and time points).

Two review authors will extract outcomes independently and compare and agree on the outcomes extracted. Disagreement will be resolved through discussion with a third reviewer.

\section{Data analysis and presentation}

Studies will be divided into those for prevention and those for treatment of GDM. Outcomes measured in each study will be displayed. Then, outcomes will be further grouped under three major domains: maternal outcomes, offspring outcomes (to include neonatal, infant, child and adult outcomes) and other outcomes (eg, health economic consequences). 


\section{e-Delphi survey}

A e-Delphi survey consisting of a series of rounds of data collection and analysis to reconcile the opinions of participants into a group consensus will be conducted. We will conduct a three-round, online e-Delphi survey. Custer et al (1999) have recommended that three iterations are sufficient to collect necessary information to reach a consensus. $^{25}$

Participants will be provided with the objectives of the study and asked for their consent. Participants who agree to participate in the study will be asked to score each of the outcomes contained within the two lists (GDM prevention and treatment) using a a 9-point Likert scoring scale with 1 to 3 labeled 'not important', 4 to 6 'important but not critical' and 7 to 9 'critical'. An option of 'unable to score' will also be included. This system is recommended by the Grading of Recommendations Assessment, Development and Evaluation working group for assessing the level of importance about research evidence. ${ }^{26}$ This scale has been adopted in other core outcome development research groups using the Delphi method. The questionnaire will be completed online using online survey design software and data collected will be downloaded and maintained on a secure drive.

\section{Research participants}

We have identified three broad key stakeholder groups who will be invited to participate in the e-Delphi survey and in the later consensus meeting:

1. Pregnant women at risk of GDM, with GDM or women with a previous history of GDM.

2. Healthcare professionals who care for women with GDM including obstetricians, endocrinologists, general practitioners, midwives, nurse specialists, paediatrics, neonatologist, pharmacists, physiotherapists and dieticians.

3. Researchers and policy makers with an active interest in the prevention and treatment of GDM.

All participants will be 18 years or older.

\section{Sample size and recruitment}

There is an absence of guidance on optimal sample sizes for e-Delphi surveys, but our intention is to recruit as many representatives as possible from each stakeholder group from as many countries as possible. The survey will be presented in English and therefore only those who can complete the survey in English will be able to participate. We will aim for a minimum of 20 participants from each stakeholder group in round 1 .

\section{Process for recruiting participants}

Potential participants will be recruited via email from a wide range of clinical, health and community settings. Recruitment will include listed groups of diabetes services users (eg, Diabetes Ireland) accessed via the electronic discussion email list manager. The manager will be emailed the survey information with a request to distribute an invitation email to members on their email lists. The list managers will have an opportunity to contact the researcher directly to clarify any issues or to seek further information about the survey and the research before making a decision. The distribution of the survey will be at the discretion of the email list manager. Clinicians and researchers will be invited from specialist centres globally and through the International Diabetes in Pregnancy Study Group and Diabetic Pregnancy Study Group). These clinical leads will be invited to participate by email and will be asked to forward the study details to colleagues and patient representatives who meet the inclusion criteria. Snowball sampling will be used, whereby the participants will be asked to forward the invitation to others whom they regard as having the required expertise. We aim to recruit participants from a minimum of 20 countries.

Detailed participant information will be included on the invitation email. This information will include an outline on the objectives of the study, the stakeholder groups, the estimated length of the survey and the round 1 survey link. After clicking on the survey link, participants will be again presented with the study information on the survey homepage and asked the following question to obtain consent if you are happy to participate in this study, please click 'yes' to continue'. Participants who agreed to participate will be encouraged to complete the whole e-Delphi process; yet, they are free to withdraw from the study at any point.

To reduce the attrition rate, the importance of completing all three rounds will be emphasised and up to two reminder emails will be sent after each round launch with details of current response rates. If a small number of responders is observed in one or more of the three stakeholder groups, the e-Delphi protocol for subsequent rounds will be reviewed and revised. If a low number of responders is observed in one stakeholder group, we will consider grouping them with another stakeholder group if it is appropriate to do so and after consultation with members of the SAG. Attrition bias will be examined between rounds to ensure that participants staying in have scored outcomes similar to those leaving the study, thereby having no impact on the final results.

\section{Round 1 e-Delphi study}

The round 1 instrument will request participant details as follows: name, gender identity, country of residence and email address. Consent to participate will be obtained as described above. Participants will be asked to identify the stakeholder group that most represents their stakeholding perspective.

The two lists of outcomes generated from stage 1 will be presented and participants will be asked to rank the importance of each outcome for inclusion in the respective COS on a 9-point Likert scale from 1 to 9 wherein 1 signifies 'not important' and 9 signifies 'critical'. This scale has been used previously in the development of a number of COSs. ${ }^{17} 21$ These outcomes will be ordered 
alphabetically to avoid weighting of outcomes caused by the order in which they are displayed.

An open question will be placed at the end of the questionnaire where participants can include up to two additional outcomes for GDM prevention studies and two additional outcomes for GDM treatment studies that were not captured in the list presented. The respondents will be asked only to suggest additional outcomes if they believe they are essential to for inclusion in the final COSs. This may identify outcomes associated with the most recent treatments available or the most pressing current concerns for stakeholders that the literature did not include. Any additional outcome listed by two or more participants will be reviewed by two researchers and will be included for rating in round 2 if the additional outcome represents a de novo outcome. The need for more than two participants will be reviewed if recruitment is lower than expected.

The results of round 1 will be analysed using descriptive statistics, including the proportion of each stakeholding group scoring for each rating point on the Likert scale (ie, for each point from 1 to 9 ). The number of participants taking part will be recorded. Participants will be asked to complete the survey within a time scale, which will be informed by a pilot study as outlined below. Up to two reminder emails will be sent after the first email to reduce the attrition rate.

Where any survey is incomplete, the respondent will be contacted to encourage completion. The survey will be written in plain language terms, developed in conjunction with the SAG to facilitate patient understanding. All participants will complete the same survey. Those who do not respond to the invitation email will not be further contacted.

\section{Pilot study}

We will pilot the first round questionnaire to ensure that it is practical and easy to use and to decide on the time taken for participants to complete a round of the e-Delphi survey.

\section{Round 2 e-Delphi study}

Only participants who completed the questionnaire in round 1 will be invited to participate in round 2. Each participant will be provided with his/her individual ratings for each outcome in round 1 and with the distribution of scores of each outcome from the three stakeholder groups separately. They will then be asked to provide their name and email and re-rate each outcome with consideration of the insights of other participants from the three stakeholder groups. They will have the opportunity to change their response from the previous round.

The results of round 2 will be summarised using descriptive statistics. The number of participants taking part will be recorded. For each outcome, the distribution of scores for each point in the Likert scale will be calculated for each stakeholder group. Consistent with other studies using the Delphi approach, prespecified criteria, 'consensus criteria', will be used for outcomes to be retained or dropped at the end of this round. Each outcome will be classified as 'consensus in', 'consensus out' or 'no consensus' according to the classification in table 1. All outcomes scored as 'consensus in' will be carried forward to round 3.

\section{Round 3 e-Delphi study}

The round 3 questionnaire will be forwarded to participants who completed rounds 1 and 2. As in round 2, each participant will be provided with his/her individual ratings for each outcome in round 2 and with the proportion of scores of each outcome from the three stakeholder groups separately alongside his/her own score from round 2. Participants will be asked to provide their name and email and re-rate each outcome again as in round 2.

The results of round 3 will be summarised using descriptive statistics. The number of participants taking part will be recorded. For each outcome, the distribution of scores for each point in the Likert scale will be recorded. Each outcome will be classified as 'consensus in', 'consensus out' or 'no consensus' according to the classification in table 1. All outcomes scored as 'consensus in' or 'no consensus' will be carried forward to the consensus meeting.

\section{Consensus meeting}

A consensus face-to-face meeting will be held with the representatives from each of the three stakeholder groups at a location agreed by the SAG. We anticipate a total number of 15-20 representatives and will aim to include representatives from different countries and at least five representatives from each of the stakeholder groups. The meeting will be facilitated by an experienced, non-voting chair facilitator. Results from the e-Delphi will be presented. Participants will have the opportunity to voice their opinion on whether an item should be excluded or included if the outcome reached was 'consensus in' or 'consensus out'; and where

Table 1 e-Delphi consensus definition

\begin{tabular}{lll} 
Consensus in & $\begin{array}{l}\text { Consensus that outcome should be } \\
\text { included in the core outcome set (COS) }\end{array}$ & $\begin{array}{l}\geq 70 \% \text { participants scoring } 7 \text { to } 9 \text { AND }<15 \% \\
\text { participants scoring } 1 \text { to } 3\end{array}$ \\
Consensus out & $\begin{array}{l}\text { Consensus that outcome should not be } \\
\text { included in the COS }\end{array}$ & $\begin{array}{l}\leq 50 \% \text { participants scoring } 7-9 \text { in each } \\
\text { stakeholder group }\end{array}$ \\
No consensus & $\begin{array}{l}\text { Uncertainty about importance of the } \\
\text { outcome }\end{array}$ & Anything else \\
\hline
\end{tabular}


outcomes reached 'no consensus', further discussion will take place. Outcomes may be combined based on this group discussion.

Following the discussion on each outcome, participants will be asked to anonymously vote for inclusion or exclusion and 'consensus in criteria' from table 1 will be used to decide if an outcome is included. At the end of the meeting, the recommended lists of outcomes will be reviewed and a consensus document with the two COSs will be drafted following completion of the consensus meeting.

\section{Identifying existing work}

WOMen and Babies health and wellbeing: Action through Trials initiative has developed 'standard outcomes' covering prevention, detection, management and follow-up in GDM research. A survey completed after the development of these standard outcomes revealed significant discrepancies in outcomes prespecified in reviews and reported by randomised trials with the authors calling for the development of a COS to reduce research waste and improve health outcomes. ${ }^{27}$ This current study will include a broad range of views in the methodology and will fulfil the specific focus on developing COSs in the realm of GDM.

The final COSs will define 'what is to be collected' and not 'how it is to be collected'. In the field of diabetes and pregnancy, there exists a previously published repository of definitions that may be referenced and we anticipate publishing definitions from this repository alongside the final COSs where possible. ${ }^{28}$

The final COS will be reported in compliance with COS-STAR recommendations. ${ }^{29}$

One of the challenges associated with development of COS will be ensuring that other research groups take up its use. Presenting this project at scientific conferences and scheduling additional meetings of researchers in the research area of GDM at national and international conferences will further increase the uptake of the proposed COSs. In addition, the authors will actively engage with the Cochrane Pregnancy and Childbirth Group to promote awareness of the COSs.

While we will make an effort to ensure that representatives from low and middle income countries are invited to participate in each stage of the COS development, this has been a challenge in previous COS studies and may limit the relevance of the final COSs to these countries. In addition, our literature search, e-Delphi and consensus meetings will be limited to the English language, which may exacerbate this issue. In keeping with available resources, we have not planned a qualitative dimension to our study, which could have the potential to enhance this work. While we acknowledge that this is not the same as qualitative research, we will give the opportunity to representatives within the SAG to provide feedback on the lists of outcomes. This approach is suggested by COMET as an alternative to qualitative research. ${ }^{21}$

\section{CONCLUSION}

It is anticipated that the proposed COSs will provide an evidence-based and consensus-driven guidance for future GDM trials that evaluate interventions for the prevention and treatment of GDM.

\section{Author affiliations}

${ }^{1}$ Department of Endocrinology, Mayo Clinic Rochester, Rochester, Minnesota, USA ${ }^{2}$ College of Medicine, Nursing and Health Sciences, National University of Ireland Galway, Galway, Ireland

${ }^{3}$ Evidence Synthesis Ireland, National University of Ireland Galway, Galway, Ireland ${ }^{4}$ Liggins Institute, University of Aukland, Auckland, New Zealand

${ }^{5}$ Department of Paediatrics and Child Health, University College Cork, Cork, Ireland

${ }^{6}$ Women's Health Research Unit, Queen Mary University of London, London, UK

${ }^{7}$ Trials Methodology Research Network, Health Research Board of Ireland, Galway, Ireland

\section{Twitter Aoife Maria Egan @egan_am}

Contributors FD and DD conceived the study; NF and AME developed the protocol and drafted the initial manuscript; LB, DB, CC, ED and ST contributed to the study design, critically reviewed and revised the protocol drafts. All authors agreed on the final draft manuscript for submission and are accountable for all aspects of the work.

Funding The authors have not declared a specific grant for this research from any funding agency in the public, commercial or not-for-profit sectors.

\section{Competing interests None declared.}

Patient consent for publication Not required.

Ethics approval Ethical approval to conduct this study was obtained from the ethics committee at Galway University Hospitals on 13 December 2018 (Reference: C.A.2078).

Provenance and peer review Not commissioned; externally peer reviewed.

Open access This is an open access article distributed in accordance with the Creative Commons Attribution Non Commercial (CC BY-NC 4.0) license, which permits others to distribute, remix, adapt, build upon this work non-commercially, and license their derivative works on different terms, provided the original work is properly cited, appropriate credit is given, any changes made indicated, and the use is non-commercial. See: http://creativecommons.org/licenses/by-nc/4.0/.

ORCID iD

Aoife Maria Egan http://orcid.org/0000-0003-0379-9279

\section{REFERENCES}

1 Kim C. Gestational diabetes: risks, management, and treatment options. Int J Womens Health 2010;2:339-51.

2 Guariguata L, Linnenkamp U, Beagley J, et al. Global estimates of the prevalence of hyperglycaemia in pregnancy. Diabetes Res Clin Pract 2014;103:176-85.

3 Cho NH, Shaw JE, Karuranga S, et al. IDF diabetes atlas: global estimates of diabetes prevalence for 2017 and projections for 2045. Diabetes Res Clin Pract 2018;138:271-81.

4 Damm P, Houshmand-Oeregaard A, Kelstrup L, et al. Gestational diabetes mellitus and long-term consequences for mother and offspring: a view from Denmark. Diabetologia 2016;59:1396-9.

5 Dall TM, Zhang Y, Chen YJ, et al. The economic burden of diabetes. Health Aff 2010;29:297-303.

6 Tobias DK, Hu FB, Forman JP, et al. Increased risk of hypertension after gestational diabetes mellitus: findings from a large prospective cohort study. Diabetes Care 2011;34:1582-4.

7 Shoham I, Wiznitzer A, Silberstein T, et al. Gestational diabetes complicated by hydramnios was not associated with increased risk of perinatal morbidity and mortality. Eur J Obstet Gynecol Reprod Biol 2001;100:46-9.

8 Gorgal R, Gonçalves E, Barros M, et al. Gestational diabetes mellitus: a risk factor for non-elective cesarean section. J Obstet Gynaecol Res 2012;38:154-9.

9 Bellamy L, Casas J-P, Hingorani AD, et al. Type 2 diabetes mellitus after gestational diabetes: a systematic review and meta-analysis. The Lancet 2009;373:1773-9. 
10 Lowe WL, Scholtens DM, Lowe LP, et al. Association of gestational diabetes with maternal disorders of glucose metabolism and childhood adiposity. JAMA 2018;320:1005-16.

11 Kc K, Shakya S, Zhang H. Gestational diabetes mellitus and macrosomia: a literature review. Ann Nutr Metab 2015;66 Supp 2:14-20.

12 Al-Khalifah R, Al-Subaihin A, Al-Kharfi T, et al. Neonatal short-term outcomes of gestational diabetes mellitus in Saudi mothers: a retrospective cohort study. J Clin Neonatol 2012;1:29-33.

13 Langer O, Conway D, McFarland M, et al. Shoulder dystocia and birth trauma in GDM and PEDM: a seven-year experience. Am J Obstet Gynecol 1997;176:S138.

14 American Diabetes Association. Gestational diabetes mellitus. Diabetes Care 2000;23 Suppl 1:S77-9.

15 Brown J, Alwan NA, West J, et al. Lifestyle interventions for the treatment of women with gestational diabetes. Cochrane Database Syst Rev 2017;107.

16 Bennett WL, Robinson KA, Saldanha IJ, et al. High priority research needs for gestational diabetes mellitus. J Womens Health 2012;21:925-32.

17 van Tol RR, Melenhorst J, Dirksen CD, et al. Protocol for the development of a core outcome set (COS) for hemorrhoidal disease: an international Delphi study. Int J Colorectal Dis 2017;32:1091-4.

18 Brown J, Martis R, Hughes B, et al. Oral anti-diabetic pharmacological therapies for the treatment of women with gestational diabetes. Cochrane Database Syst Rev 2017;33.

19 Casey BM, Duryea EL, Abbassi-Ghanavati M, et al. Glyburide in women with mild gestational diabetes. Obstetrics \& Gynecology 2015;126:303-9.
20 Hui AL, Sevenhuysen G, Harvey D, et al. Barriers and coping strategies of women with gestational diabetes to follow dietary advice. Women Birth 2014;27:292-7.

21 Williamson PR, Altman DG, Bagley H, et al. The COMET Handbook: version 1.0. Trials 2017:18:280.

22 Kirkham JJ, Davis K, Altman DG, et al. Core outcome Set-STAndards for development: the COS-STAD recommendations. PLoS Med 2017; $14:$ e1002447

23 CROWN. Core outcomes in women's and newborn health (CROWN). Available: http://www.crown-initiative.org/journals/ [Accessed 12 Nov 2018].

24 Avalos GE, Owens LA, Dunne F, et al. Applying current screening tools for gestational diabetes mellitus to a European population: is it time for change? Diabetes Care 2013;36:3040-4.

25 Custer RL, Scarcella JA, Stewart BR. The Modified Delphi Technique - A Rotational Modification. Journal of Career and Technical Education 1999;15:50-8.

26 Williamson PR, Altman DG, Blazeby JM, et al. Developing core outcome sets for clinical trials: issues to consider. Trials 2012;13:132.

27 Bain E, Middleton P, Crowther CA. Progressing towards standard outcomes in gestational diabetes Cochrane reviews and randomised trials. Aust N Z J Obstet Gynaecol 2016;56:113-6.

28 Feig DS, Corcoy R, Jensen DM, et al. Diabetes in pregnancy outcomes: a systematic review and proposed codification of definitions. Diabetes Metab Res Rev 2015;31:680-90.

29 Kirkham JJ, Gorst S, Altman DG, et al. Core outcome SetSTAndards for reporting: the COS-STAR statement. PLoS Med 2016;13:e1002148. 